PROCEEDINGS OF THE

AMERICAN MATHEMATICAL SOCIETY

Volume 140, Number 11, November 2012, Pages 3823-3830

S 0002-9939(2012)11244-4

Article electronically published on March 20, 2012

\title{
SOLVABLE COMPLEMENTED LIE ALGEBRAS
}

\author{
DAVID A. TOWERS
}

(Communicated by Kailash C. Misra)

\begin{abstract}
In this paper a characterisation is given of solvable complemented Lie algebras. They decompose as a vector space direct sum of abelian subalgebras, and their ideals relate nicely to this decomposition. The class of such algebras is shown to be a formation whose residual is the ideal closure of the prefrattini subalgebras.
\end{abstract}

\section{Prefrattini subalgebras}

Throughout, $L$ will denote a finite-dimensional solvable Lie algebra over a field $F$. We define the nilpotent residual, $L^{\infty}$, of $L$ to be the smallest ideal of $L$ such that $L / L^{\infty}$ is nilpotent. Clearly this is the intersection of the terms of the lower central series for $L$. The derived series for $L$ is the sequence of ideals $L^{(i)}$ of $L$ defined by $L^{(0)}=L, L^{(i+1)}=\left[L^{(i)}, L^{(i)}\right]$ for $i \geq 0$; we will also write $L^{2}$ for $L^{(1)}$. If $L^{(n)}=0$ but $L^{(n-1)} \neq 0$ we say that $L$ has derived length $n$. We say that $L$ is completely solvable if $L^{2}$ is nilpotent. Algebra direct sums will be denoted by $\oplus$, whereas vector space direct sums will be denoted by $\dot{+}$.

The Frattini subalgebra of $L, \phi(L)$, is the intersection of the maximal subalgebras of $L$. When $L$ is solvable this is always an ideal of $L$, by [1, Lemma 3.4]. For a subalgebra $U$ of $L$ we denote by $[U: L]$ the set of all subalgebras $S$ of $L$ with $U \subseteq S \subseteq L$. We say that $[U: L]$ is complemented if, for any $S \in[U: L]$ there is a $T \in[U: L]$ such that $S \cap T=U$ and $\langle S, T\rangle=L$. We denote by $[U: L]_{\max }$ the set of maximal subalgebras in $[U: L]$, that is, the set of maximal subalgebras of $L$ containing $U$.

Let

$$
0=A_{0} \subset A_{1} \subset \ldots \subset A_{n}=L
$$

be a chief series for $L$. We say that $A_{i} / A_{i-1}$ is a Frattini chief factor if $A_{i} / A_{i-1} \subseteq$ $\phi\left(L / A_{i-1}\right)$; it is complemented if there is a maximal subalgebra $M$ of $L$ such that $L=A_{i}+M$ and $A_{i} \cap M=A_{i-1}$. When $L$ is solvable it is easy to see that a chief factor is Frattini if and only if it is not complemented.

We define the set $\mathcal{I}$ by $i \in \mathcal{I}$ if and only if $A_{i} / A_{i-1}$ is not a Frattini chief factor of $L$. For each $i \in \mathcal{I}$ put

$$
\mathcal{M}_{i}=\left\{M \in\left[A_{i-1}, L\right]_{\max }: A_{i} \not \subseteq M\right\} .
$$

Received by the editors April 20, 2011 and, in revised form, April 26, 2011 and May 8, 2011. 2010 Mathematics Subject Classification. Primary 17B05, 17B30; Secondary 17B10, 17B50.

Key words and phrases. Lie algebras, complemented, solvable, Frattini ideal, prefrattini subalgebra, residual, Lie $A$-algebra.

(C)2012 American Mathematical Society Reverts to public domain 28 years from publication 
Then $B$ is a prefrattini subalgebra of $L$ if

$$
B=\bigcap_{i \in \mathcal{I}} M_{i} \text { for some } M_{i} \in \mathcal{M}_{i} .
$$

It was shown in 7 that the definition of prefrattini subalgebras does not depend on the choice of chief series.

The subalgebra $B$ avoids $A_{i} / A_{i-1}$ if $B \cap A_{i}=B \cap A_{i-1}$; likewise, $B$ covers $A_{i} / A_{i-1}$ if $B+A_{i}=B+A_{i-1}$. Let $\Pi(L)$ be the set of prefrattini subalgebras of $L$. Then the following results were established in 7 .

Theorem 1.1. Let $L$ be a solvable Lie algebra over a field $F$.

(i) If $B$ is a prefrattini subalgebra of $L$, then it covers all Frattini chief factors of $L$ and avoids the rest.

(ii) If $B$ is a prefrattini subalgebra of $L$, then

$$
\operatorname{dim} B=\sum_{i \notin \mathcal{I}}\left(\operatorname{dim} A_{i}-\operatorname{dim} A_{i-1}\right) ;
$$

in particular, all prefrattini subalgebras of $L$ have the same dimension.

(iii) If $A$ is an ideal of $L$ and $S \in \Pi(L)$, then $(S+A) / A \in \Pi(L / A)$.

(iv) $\phi(L)=\bigcap_{B \in \Pi(L)} B$.

(v) If $L$ is completely solvable, then $\Pi(L)=\{\phi(L)\}$.

(vi) Suppose that $F$ has characteristic $p$ and that $L^{\infty}$ has nilpotency class less than $p$. Then the elements of $\Pi(L)$ are conjugate under inner automorphisms of the form $\exp (\operatorname{ad} x)$ with $x \in L^{\infty}$.

(vii) $L$ is complemented if and only if $\Pi(L)=\{0\}$.

If $L^{2}$ is not nilpotent, then $\Pi(L)$ can contain more than one element (see [7]).

\section{THE PREFRATTINI RESIDUAL}

Here we use the ideas of the previous section to re-examine complemented Lie algebras, that is, Lie algebras $L$ for which $[0: L]$ is complemented, as studied in [5]. Results for groups similar to those in the next theorem were stated by Bechtell in [2].

Theorem 2.1. Let $L$ be a solvable Lie algebra over any field $F$. Then the following are equivalent:

(i) $L$ is complemented.

(ii) The prefrattini subalgebras of $L$ are all trivial.

(iii) $L$ and all of its epimorphic images are $\phi$-free.

(iv) L splits over all of its ideals.

Proof. (i) $\Rightarrow$ (ii) : If $L$ is complemented, then $\Pi(L)=\{0\}$, by Theorem 1.1 (vii).

(ii) $\Rightarrow$ (iii) : Suppose that $\Pi(L)=\{0\}$, let $L / B$ be any epimorphic image of $L$, and suppose that $\phi(L / B) \neq 0$. Then there is a Frattini chief factor of $L, C / B$, contained in $\phi(L / B)$. But now any prefrattini subalgebra has dimension greater than or equal to $\operatorname{dim}(C / B)$, by Theorem 1.1 (ii), a contradiction. This establishes (iii).

(iii) $\Rightarrow$ (iv) : Suppose that $L$ and all of its epimorphic images are $\phi$-free. We use induction on $\operatorname{dim} L$. The result is clear if $\operatorname{dim} L=1$. So suppose it holds for Lie algebras of dimension less than $\operatorname{dim} L$, and let $B$ be a non-trivial ideal of $L$. If $B$ is a minimal ideal of $L$, then the result follows from [3, Lemma 7.2]. If $B$ is not 
a minimal ideal, let $A$ be a minimal ideal of $L$ contained in $B$. Then $L / A$ splits over $B / A$ by the inductive hypothesis. Thus there is a subalgebra $C$ of $L$ with $A \subseteq C$ such that $L=B+C$ and $B \cap C=A$. Moreover, there is a subalgebra $M$ of $L$ such that $L=A \dot{+} M$ by [3, Lemma 7.2]. But now $C=A \dot{+}(M \cap C)$, whence $L=B \dot{+}(M \cap C)$, and (iv) is established.

(iv) $\Rightarrow$ (i) : Suppose that $L$ splits over all of its ideals. We use induction on $\operatorname{dim} L$ again. The result is clear if $\operatorname{dim} L=1$. So suppose it holds for Lie algebras of dimension less than $\operatorname{dim} L$, and let $A$ be a minimal ideal of $L$. Then $L=A \dot{+} M$ for some subalgebra $M$ of $L$. It is clear that $M \cong L / A$ splits over all of its ideals and so is complemented by the inductive hypothesis. It follows from [5, Lemma 4] that $L$ is complemented.

We say that $L$ is elementary if $\phi(B)=0$ for every subalgebra $B$ of $L$. Let $A$ be a vector space of finite dimension and let $B$ be an abelian completely reducible subalgebra of $\operatorname{gl}(A)$. It was shown in [8, Proposition 2.4] that the semidirect product $A \rtimes B$ is an elementary Lie algebra; we call such an algebra an elementary Lie algebra of type $I$. Then we have the following characterisation of completely solvable complemented Lie algebras.

Theorem 2.2. Let $L$ be a completely solvable Lie algebra. Then the following are equivalent:

(i) $L$ is complemented;

(ii) $\phi(L)=0$;

(iii) $L$ is elementary;

(iv) $L \cong A \oplus E$, where $A$ is an abelian Lie algebra and $E$ is an elementary Lie algebra of type $I$.

Proof. The equivalence of (i), (ii) and (iii) is [5, Theorem 1]. The equivalence of (iv) follows from [8, Theorem 2.5] (the requirement of a perfect field in that result is required only to establish that an elementary algebra is completely solvable, and that is not needed here).

Lemma 2.3. Let $L$ be a solvable Lie algebra, let $B, C$ be ideals of $L$ with $B \cap C=0$, and suppose that $L / B$ and $L / C$ are complemented. Then $L$ splits over $B$ and over $C$.

Proof. We show that $L$ splits over $C$. Since $L / B$ is complemented there is a subalgebra $U$ of $L$ with $B \subseteq U$ such that $L=(B+C)+U=C+U$ and $(B+C) \cap U=B$. Hence $C \cap U \subseteq C \cap(B+C) \cap U=C \cap B=0$.

A class $\mathcal{H}$ of finite-dimensional solvable Lie algebras is called a homomorph if it contains, along with an algebra $L$, all epimorphic images of $L$. A homomorph $\mathcal{H}$ is called a formation if $L / M, L / N \in \mathcal{H}$, where $M, N$ are ideals of $L$, implies that $L / M \cap N \in \mathcal{H}$. If $\mathcal{H}$ is a formation, then for every solvable Lie algebra $L$ there is a smallest ideal $R$ such that $L / R \in \mathcal{H}$; this is called the $\mathcal{H}$-residual of $L$. We denote the class of solvable complemented Lie algebras by $\mathcal{C}$. Then we have the following result.

Theorem 2.4. $\mathcal{C}$ is a formation.

Proof. First note that $\mathcal{C}$ is a homomorph, by [5, Lemma 3]. Let $B, C$ be distinct ideals of $L$ with $L / B, L / C \in \mathcal{C}$. We need to show that $L / B \cap C \in \mathcal{C}$. Without loss 
of generality we may suppose that $B \cap C=0$. Let $0<B_{k}<\ldots<B_{1}=B$ be part of a chief series for $L$. We use induction on $k$. If $k=1$, then $B$ is a minimal ideal of $L$ and the result follows from Lemma 2.3] and [5, Lemma 4]. So suppose it holds whenever $k<n$ and that we have $k=n$. Then $B / B_{n},\left(C+B_{n}\right) / B_{n}$ are distinct ideals of $L / B_{n}$ and the corresponding factor algebras are isomorphic to $L / B$ and $(L / C) /\left(\left(C+B_{n}\right) / C\right)$ respectively. These are both complemented (by [5, Lemma 3] in the case of the second). It follows from the inductive hypothesis that $L / B_{n}$ is complemented. But now $L$ is complemented by Lemma 2.3 and [5, Lemma 4], and the result follows.

We define the prefrattini residual of a solvable Lie algebra $L$ to be

$$
\pi(L)=\bigcap\{B: B \text { is an ideal of } L \text { and } L / B \in \mathcal{C}\} .
$$

Clearly $\pi(L)$ is the smallest ideal of $L$ such that $L / \pi(L) \in \mathcal{C}$. It is also the ideal closure of the prefrattini subalgebras of $L$, by Theorem 2.1 .

The class of solvable elementary Lie algebras is also a formation whose residual is the elementary commutator, $E(L)$ (see [4, Theorem 5.1]). The abelian socle of $L$, Asoc $L$, is the sum of the minimal abelian ideals of $L$. We have the following properties of $\pi(L)$.

Theorem 2.5. Let $L$ be a solvable Lie algebra. Then:

(i) $\phi(L) \subseteq \pi(L) \subseteq E(L)$; if $L$ is completely solvable, then $\phi(L)=\pi(L)=$ $E(L)$.

(ii) If $A$ is an ideal of $L$, then $\pi(L / A)=(\pi(L)+A) / A$; in particular, $\pi(L / \phi(L))$ $=\pi(L) / \phi(L)$.

(iii) $\pi(L)$ is nilpotent if and only if $\pi(L)=\phi(L)$.

(iv) If $B$ is a prefrattini subalgebra of $L$, then $\pi(L)=B+\pi(L)^{\infty}$.

Proof. (i) This follows from Theorem 2.1 and Theorem 2.2 .

(ii) Let $A$ be an ideal of $L$ and put $\pi(L / A)=P / A$. Then we have that $L / P \cong$ $(L / A) /(P / A)$ is complemented. Hence $\pi(L)+A \subseteq P$. Also $(L / A) /((\pi(L)+A) / A) \cong$ $(L / \pi(L)) /((\pi(L)+A) / \pi(L))$ is complemented, by [5, Lemma 3], so $P \subseteq \pi(L)+A$ and the result follows.

(iii) If $\pi(L)=\phi(L)$, then $\pi(L)$ is nilpotent, by [3. Theorem 6.1]. Conversely, let $\pi(L)$ be nilpotent. By (i), we need only prove that $\pi(L) \subseteq \phi(L)$. By (ii), we can work in $L / \phi(L)$, so we may suppose that $\phi(L)=0$. Let $N$ be the nilradical of $L$ and let $B$ be a prefrattini subalgebra of $L$. Then $N=\operatorname{Asoc} L=A_{1} \oplus \ldots \oplus A_{n}$, where $A_{i}$ is a minimal ideal of $L$ for $1 \leq i \leq n$, and $L=N \dot{+} C$ for some subalgebra $C$ of $L$, by [3, Theorems 7.3, 7.4]. Then $\left(A_{1} \oplus \ldots \oplus A_{i+1}\right) /\left(A_{1} \oplus \ldots \oplus A_{i}\right)$ is a complemented chief factor of $L$ for each $1 \leq i \leq n-1$, and so is avoided by $B$. It follows that $B \cap N=0$. But then $\pi(L)=0$ and the converse follows from (ii).

(iv) We have that $\left(B+\pi(L)^{\infty}\right) / \pi(L)^{\infty}$ is a prefrattini subalgebra of $L / \pi(L)^{\infty}$ by Theorem 1.1 (iii). Moreover, $\pi\left(L / \pi(L)^{\infty}\right)=\pi(L) / \pi(L)^{\infty}$, by (ii) above, and this is nilpotent. It follows from (iii) that $\pi\left(L / \pi(L)^{\infty}\right)=\phi\left(L / \pi(L)^{\infty}\right) \subseteq(B+$ $\left.\pi(L)^{\infty}\right) / \pi(L)^{\infty}$, by Theorem [1.1 (iv), whence $\pi(L) \subseteq B+\pi(L)^{\infty}$. The reverse inclusion is clear.

We define the nilpotent series for $L$ inductively by $N_{0}(L)=0, N_{i+1} / N_{i}=$ $N\left(L / N_{i}(L)\right.$ for $i>0$, where $N(L)$ denotes the nilradical of $L$. Finally we have the following characterisation of solvable complemented Lie algebras, which is analogous to a result of Zacher for groups (see [10]). 
Theorem 2.6. The solvable Lie algebra $L$ is complemented if and only if $\phi\left(L / N_{i}(L)\right)=0$ for all $i \geq 0$.

Proof. Suppose first that $L$ is complemented. Then $L / N_{i}(L)$ is complemented, by [5. Lemma 3], and so $\phi\left(L / N_{i}(L)\right)=0$, by Theorem 2.1.

Suppose conversely that $\phi\left(L / N_{i}(L)\right)=0$ for all $i \geq 0$. We use induction on $\operatorname{dim} L$. The result is clear if $\operatorname{dim} L=1$, so suppose the result holds for all solvable Lie algebras of dimension less than that of $L$. Then $L / N(L)$ is complemented, by the inductive hypothesis. Moreover, we have that $L=N(L) \dot{+} B$ for some subalgebra $B$ of $L$, and $N(L)=$ Asoc $L$, by [3, Theorems 7.3, 7.4]. Put Asoc $L=A_{1} \oplus \ldots \oplus A_{n}$. If $n=1$, then $L$ splits over $A_{1}$ and $L / A_{1}$ is complemented, so $L$ is complemented, by [5, Lemma 4]. So suppose that $n>1$ and put $C_{i}=A_{1} \oplus \ldots \oplus \hat{A}_{i} \oplus \ldots \oplus$ $A_{n}$, where $\hat{A}_{i}$ means that $A_{i}$ is missing from the direct sum. Then $L / C_{i}$ splits over Asoc $L / C_{i}$ and $\left(L / C_{i}\right) /\left(\operatorname{Asoc} L / C_{i}\right) \cong L / N(L)$ is complemented, so $L / C_{i}$ is complemented, by [5, Lemma 4] again. It follows from Theorem 2.4 that $L \cong$ $L / \bigcap_{i=1}^{n} C_{i}$ is complemented.

A consequence of the corresponding result for groups is that every normal subgroup of a complemented solvable group is itself complemented. The analogue of this holds for completely solvable Lie algebras, by Theorem 2.2. However, the analogue does not hold for all solvable Lie algebras, as the following example shows.

Example 2.7. Let $F$ be a field of characteristic $p$ and consider the Lie algebra $L=$ $\left(\bigoplus_{i=0}^{p-1} F e_{i}\right) \dot{+} F x \dot{+} F y$ with $\left[e_{i}, x\right]=e_{i+1}$ for $i=0, \ldots, p-2,\left[e_{p-1}, x\right]=e_{0},\left[e_{i}, y\right]=$ $i e_{i}$ for $i=0, \ldots, p-1,[x, y]=x$, and all other products zero. Then $A=\bigoplus_{i=0}^{p-1} F e_{i}$ is the unique minimal ideal of $L, L$ splits over $A$ and $L / A$ is two-dimensional and so complemented. It follows from [5, Lemma 4] that $L$ is complemented. However, $B=A \dot{+} F x=L^{2}$ is an ideal of $L$, and $\phi(B)=F\left(x_{0}+\cdots+x_{p-1}\right)$, so $B$ is not complemented.

\section{DeComposition ReSUlts For COMPLEMEnted Algebras}

A Lie algebra $L$ is called an $A$-algebra if every nilpotent subalgebra of $L$ is abelian. Here we have some basic structure theorems which mirror those obtained for solvable Lie $A$-algebras in [6]. Where proofs are very similar to the corresponding one in [6] we will sketch the proof for the convenience of the reader and give a reference to [6] for more details. First we see that $L$ splits over the terms in its derived series. To establish this the following lemma is useful.

Lemma 3.1. Let $L$ be a metabelian Lie algebra that splits over $L^{2}$. Then $L^{2}=L^{\infty}$.

Proof. We have that $L=L^{2} \dot{+} C$, where $C$ is abelian. Then $L^{2}=\left[L^{2}, C\right]=\left[L^{2}, L\right]=$ $L^{3}$, whence $L^{2}=L^{\infty}$.

Theorem 3.2. Let $L$ be a solvable complemented Lie algebra. Then $L$ splits over each term in its derived series. Moreover, the Cartan subalgebras of $L^{(i)} / L^{(i+2)}$ are precisely the subalgebras that are complements to $L^{(i+1)} / L^{(i+2)}$ for $i \geq 0$.

Proof. The first assertion follows from Theorem 2.1(iv). The second follows easily from Lemma 3.1] and [9, Theorem 4.4.1.1].

This gives us the following characterisation of solvable complemented Lie algebras. 
Corollary 3.3. Let $L$ be a solvable Lie algebra of derived length $n+1$. Then $L$ is complemented if and only if the following hold:

(i) $L=A_{n} \dot{+} A_{n-1} \dot{+} \ldots \dot{+} A_{0}$ where $A_{i}$ is an abelian subalgebra of $L$ for each $0 \leq i \leq n$

(ii) $L^{(i)}=A_{n} \dot{+} A_{n-1} \dot{+} \ldots \dot{+} A_{i}$ for each $0 \leq i \leq n$;

(iii) $L^{(i)} / L^{(i+1)}$ is completely reducible as an $\left(L / L^{(i+1)}\right)$-module for each $1 \leq$ $i \leq n$.

Proof. Suppose first that $L$ is complemented. By Theorem 3.2 there is a subalgebra $B_{n}$ of $L$ such that $L=L^{(n)} \dot{+} B_{n}$. Put $A_{n}=L^{(n)}$. Similarly $B_{n} \cong L / L^{(n)}$ is complemented, by [5, Lemma 3], so $B_{n}=A_{n-1} \dot{+} B_{n-1}$, where $A_{n-1}=\left(B_{n}\right)^{(n-1)}$. Continuing in this way we get (i). A straightforward induction proof shows (ii). Finally, $L^{(i)} / L^{(i+1)} \subseteq N\left(L / L^{(i+1)}\right)=\operatorname{Asoc}\left(L / L^{(i+1)}\right)$, by Theorem 2.1 (iii) and 3, Theorem 7.4], which gives (iii).

Suppose now that (i), (ii) and (iii) hold. Then $L$ is complemented by repeated use of [5, Lemma 4].

Next we aim to show the relationship between ideals of $L$ and the decomposition given in Corollary 3.3. First we need the following lemmas.

Lemma 3.4. Let $L$ be a Lie algebra. Then $Z(L) \cap L^{2} \subseteq \phi(L)$.

Proof. Let $M$ be any maximal subalgebra of $L$. If $Z(L) \nsubseteq M$, then $L=M+Z(L)$ and $L^{2} \subseteq M$.

Lemma 3.5 (cf. 6, Lemma 3.4]). Let $L$ be a solvable complemented Lie algebra of derived length $\leq n+1$, and suppose that $L=B \dot{+} C$, where $B=L^{(n)}$ and $C$ is a subalgebra of $L$. If $D$ is an ideal of $L$, then $D=(B \cap D) \dot{+}(C \cap D)$.

Proof. Let $L$ be a counterexample for which $\operatorname{dim} L+\operatorname{dim} D$ is minimal. Suppose first that $D^{2} \neq 0$. Then $D^{2}=\left(B \cap D^{2}\right) \dot{+}\left(C \cap D^{2}\right)$ by the minimality condition. Moreover, by considering $L / D^{2}$ we have that $D=B \cap D \dot{+} C \cap D$, a contradiction. We therefore have that $D^{2}=0$. Similarly, by considering $L / B \cap D$, we have that $B \cap D=0$.

Put $E=C^{(n-1)}$, so that $L^{(n-1)}=E+B$. Then $(D+B) / B$ and $(E+B) / B$ are abelian ideals, and so are inside the nilradical of the complemented Lie algebra $L / B$, which is abelian. Hence

$$
[D, E] \subseteq[D+B, E+B] \subseteq B \text { and }[D, E] \subseteq B \cap D=0 ;
$$

that is, $D \subseteq Z_{L}(E)=Z_{B}(E)+Z_{C}(E)$.

Now $L^{(n-1)}=B+E$, so $B=L^{(n)}=(B+E)^{2}=[B, E]$. Let $L^{(n-1)}=L_{0} \dot{+} L_{1}$ be the Fitting decomposition of $L^{(n-1)}$ relative to ad $E$. Then $B \subseteq L_{1}$ so that $Z_{B}(E) \subseteq$ $L_{0} \cap L_{1}=0$, whence $D \subseteq Z_{L}(E)=Z_{C}(E) \subseteq C$, and the result follows.

Theorem 3.6 (cf. 6. Theorem 3.5]). Let $L$ be a solvable complemented Lie algebra of derived length $n+1$ with nilradical $N$, and let $K$ be an ideal of $L$ and $A$ a minimal ideal of $L$. Then, with the same notation as Corollary 3.3.

(i) $K=\left(K \cap A_{n}\right) \dot{+}\left(K \cap A_{n-1}\right) \dot{+} \ldots \dot{+}\left(K \cap A_{0}\right)$;

(ii) $N=A_{n} \dot{+}\left(N \cap A_{n-1}\right) \dot{+} \ldots \dot{+}\left(N \cap A_{0}\right)$;

(iii) $Z\left(L^{(i)}\right)=N \cap A_{i}$ for each $0 \leq i \leq n$;

(iv) $A \subseteq N \cap A_{i}$ for some $0 \leq i \leq n$. 
Proof. (i) We have that $L=A_{n} \dot{+} B_{n}$, where $A_{n}=L^{(n)}$ from the proof of Corollary 3.3. It follows from Lemma 3.5 that $K=\left(K \cap A_{n}\right)+\left(K \cap B_{n}\right)$. But now $K \cap B_{n}$ is an ideal of $B_{n}$, which is complemented, so $B_{n}=A_{n-1} \dot{+} B_{n-1}$. Applying Lemma 3.5 again gives $K \cap B_{n}=\left(K \cap A_{n-1}\right) \dot{+}\left(K \cap B_{n-1}\right)$. Continuing in this way gives the required result.

(ii) This is clear from (i), since $A_{n}=L^{(n)}=N \cap A_{n}$.

(iii) We have that $L^{(i)}=L^{(i+1)} \dot{+} A_{i}$ from Corollary [3.3. and $Z\left(L^{(i)}\right) \cap L^{(i+1)} \subseteq$ $\phi\left(L^{(i)}\right)$, by Lemma 3.4, whence $Z\left(L^{(i)}\right) \cap L^{(i+1)} \subseteq \phi(L)=0$, using [3, Lemma 4.1]. It follows from (i) that

$$
Z\left(L^{(i)}\right)=\left(Z\left(L^{(i)}\right) \cap L^{(i+1)}\right)+\left(Z\left(L^{(i)}\right) \cap A_{i}\right)=Z\left(L^{(i)}\right) \cap A_{i} \subseteq N \cap A_{i} .
$$

It remains to show that $N \cap A_{i} \subseteq Z\left(L^{(i)}\right)$, that is, $\left[N \cap A_{i}, L^{(i)}\right]=0$. We use induction on the derived length of $L$. If $L$ has derived length one the result is clear. So suppose it holds for Lie algebras of derived length $\leq k$, and let $L$ have derived length $k+1$. Then $B=A_{k-1}+\cdots+A_{0} \cong L / L^{(k)}$ is a solvable complemented Lie algebra of derived length $k$, and, if $N$ is the nilradical of $L$, then $N \cap A_{i}$ is inside the nilradical of $B$ for each $0 \leq i \leq k-1$, so $\left[N \cap A_{i}, B^{(i)}\right]=0$ for $0 \leq i \leq k-1$, by the inductive hypothesis. But $\left[N \cap A_{i}, A_{k}\right]=\left[N \cap A_{i}, L^{(k)}\right] \subseteq[N, N]=0$, for $0 \leq i \leq k$, whence $\left[N \cap A_{i}, L^{(i)}\right]=\left[N \cap A_{i}, A_{k}+B^{(i)}\right]=0$ for $0 \leq i \leq k$.

(iv) We have $A \subseteq L^{(i)}, A \nsubseteq L^{(i+1)}$ for some $0 \leq i \leq n$. Now $\left[L^{(i)}, A\right] \subseteq$ $\left[L^{(i)}, L^{(i)}\right]=L^{(i+1)}$, so $\left[L^{(i)}, A\right] \neq A$. It follows that $\left[L^{(i)}, A\right]=0$, whence $A \subseteq$ $Z\left(L^{(i)}\right)=N \cap A_{i}$, by (iii).

A Lie algebra $L$ is called monolithic if it has a unique minimal ideal, called the monolith of $L$.

Corollary 3.7. Let $L$ be a solvable complemented monolithic Lie algebra of derived length $n+1$ with monolith $W$. Then $W=N=A_{n}=L^{(n)}=C_{L}(W), Z(L)=0$ and $[L, W]=W$.

Proof. First note that $N=A_{n} \dot{+} N \cap A_{n-1} \dot{+} \ldots \dot{+} N \cap A_{0}$ by Theorem 3.6(i). Moreover, $N \cap A_{i}$ is an ideal of $L$ for each $0 \leq i \leq n-1$, by Theorem 3.6(iii). But if $N \cap A_{i} \neq 0$, then $W \subseteq A_{n} \cap N \cap A_{i}=0$ if $i \neq n$. Hence $W=N=A_{n}$. Also $W=$ Asoc $L=N$, by Theorem 2.1 (iii) and [3, Theorem 7.4], and $N=C_{L}(N)$ by [6, Lemma 2.4].

Finally, if $Z(L) \neq 0$, then $W \subseteq Z(L) \cap L^{2}=0$, by Theorem 3.6 a contradiction. Hence $Z(L)=0$. It follows from this that $[L, W] \neq 0$, whence $[L, W]=W$.

Given these shared properties between the classes of solvable Lie $A$-algebras and solvable complemented Lie algebras it is natural to ask whether either class is contained in the other. This is not the case, as the following examples show.

Example 3.8. Let $L=F x+F y+F b$ with $[x, b]=x,[y, b]=y-x$, other products being zero. Then $\phi(L)=F x$, so $L$ is not complemented. However, it is an $A$ algebra. For, the two-dimensional subalgebras are of the form $F x+F(\alpha y+\beta b)$ $(\alpha, \beta \in F)$, and these are nilpotent only if $\beta=0$, and, in that case, it is abelian.

Examples of solvable complemented Lie algebras $L$ that are not $A$-algebras are a little harder to construct. In particular, if $L$ is completely solvable and complemented, then it is elementary, by Theorem 2.2, and so is an $A$-algebra. However, such algebras do exist in characteristic $p$, as is shown below. 
Example 3.9. Let $F$ be an algebraically closed field of characteristic $p$, let $L$ be the algebra described in Example 2.7 and let $C$ be a faithful completely reducible $L$-module. Since $L$ is monolithic, $C$ has a faithful irreducible submodule $B$. Let $X$ be the split extension of $B$ by $L$. Since $L$ is complemented, $X$ is complemented by [5, Lemma 4]. However, $X$ is solvable of index four and so cannot be an $A$-algebra, by Drensky's Theorem (see [6, Theorem 6.2]).

Notice that an easy extension of the above construction shows that, over an algebraically closed field, there are solvable complemented Lie algebras of arbitrary solvable index, whereas solvable Lie $A$-algebras over such a field have solvable index at most three.

\section{ACKNOWLEDGEMENT}

The author is grateful to the referee for a number of helpful suggestions.

\section{REFERENCES}

1. D.W. Barnes and H.M. Gastineau-Hills, On the theory of soluble Lie algebras, Math. Z. 106 (1968), 343-354. MR0232807 (38:1130)

2. H. Bechtell, The prefrattini residual, Proc. Amer. Math. Soc. 55 (1976), 267-270. MR0409633 $(53: 13385)$

3. D.A. Towers, A Frattini theory for algebras, Proc. London Math. Soc. (3) 27 (1973), 440-462. MR0427393 (55:427)

4. D.A. Towers, Elementary Lie algebras, J. London Math. Soc. (2) 7 (1973), 295-302. MR0376782 (51:12957)

5. D.A. Towers, On complemented Lie algebras, J. London Math. Soc. (2) 22 (1980), 63-65. MR579809 (81h:17006)

6. D.A. Towers, Solvable Lie A-algebras, J. Algebra 340 (2011), 1-12. MR2813558

7. D.A. Towers, Complements of intervals and prefrattini subalgebras of solvable Lie algebras, arXiv:1104.3774. To appear in Proc. Amer. Math. Soc.

8. D. A. Towers and V. R. Varea, Elementary Lie algebras and Lie A-algebras, J. Algebra 312 (2007), 891-901. MR.2333190 (2008f:17008)

9. D. J. Winter, Abstract Lie algebras, M.I.T. Press, Cambridge, Mass. (1972). MR0332905 (48:11230)

10. G. Zacher, Caratterizzazione dei gruppi risolubili d'ordine finito complementati, Rend. Sem. Mat. Univ. Padova 22 (1953), 113-122. MR0057878 (15:286d)

Department of Mathematics and Statistics, Lancaster University, Lancaster LA1 4YF, ENGLAND

E-mail address: d.towers@lancaster.ac.uk 